\title{
COMPLETE BASES AND WALLMAN REALCOMPACTIFICATIONS
}

\author{
JOSE L. BLASCO ${ }^{1}$
}

\begin{abstract}
We study a particular class of separating nest generated intersection rings on a Tychonoff space $X$, that we call complete bases. They are characterized by the equality $\beta(v(X, \mathscr{D}))=\omega(X, D)$ between their associated Wallman spaces. It is proven that for each separating nest generated intersection ring $\mathscr{D}$ there exists a unique complete base $\widehat{D}$ such that $v(X, \mathscr{D})=v(X, \hat{D})$. From this result we obtain a necessary and sufficient condition for the existence of a continuous extension to $v(X, D)$ of a real-valued function over $X$. Some applications of these results to certain inverse-closed subalgebras of $C(X)$ are given.
\end{abstract}

The word space will refer to Tychonoff spaces. In this paper we consider the Wallman compactification $\omega(X, \mathcal{D})$ and the Wallman realcompactification $v(X, \mathcal{D})$ associated with a given base ${ }^{2}$ on a space $X$. For definitions and basic results the reader is referred to [1], [9], [10]. We study the bases $\mathscr{D}$ that coincide with the trace on $X$ of all zero-sets in its associated space $v(X, \mathscr{D})$. These bases, that we call complete, have interesting properties. They are characterized by the relation $\beta(v(X, \mathscr{D}))=\omega(X, \mathscr{D}){ }^{3}$ For each base $\mathscr{D}$ on $X$ there exists a unique complete base $\hat{\mathscr{D}}$ such that $v(X, \mathscr{D})=v(X, \hat{\mathscr{D}})$. The base $\hat{\mathscr{D}}$ is the largest base with the above property and the smallest complete base on $X$ containing $\mathscr{D}$.

Frink [4] has shown that the real-valued functions over a space $X$ which may be continuously extended to $\omega(X, \mathscr{D})$ are those which are $\mathscr{D}$-uniformly continuous. In [3] D'Aristotle defined countable $\mathscr{D}$-uniform continuity and he showed that it is a sufficient but not a necessary condition for the existence of a continuous extension to $v(X, \mathcal{D})$ of a real-valued function over $X$. A necessary and sufficient condition has been obtained by Bentley and Naimpally in [2, Theorem 6]. We give another condition by means of the base के.

In order to provide examples of noncomplete bases, a general result

Received by the editors March 16, 1978 and, in revised form, August 11, 1978.

AMS (MOS) subject classifications (1970). Primary 54D60, 54D35.

Key words and phrases. Nest generated intersection ring, strong delta normal base, complete base, countable intersection property, $Q$-closure, $Q$-dense, algebra, $\sigma$-algebra.

${ }^{1}$ The author wishes to thank the referee for his suggestions.

${ }^{2}$ By a base on a space $X$ is meant a separating nest generated intersection ring on $X$ (A. K. Steiner and E. F. Steiner [10]). R. A. Alò and H. L. Shapiro [1] use the term strong delta normal base.

${ }^{3}$ Two extensions $T_{1}$ and $T_{2}$ of a space $X$ are said to be equivalent if they are homeomorphic via a map that leaves $X$ pointwise fixed. In this case we write $T_{1}=T_{2}$. 
(Theorem 4) is proven. From this result we derive that the $\sigma$-algebra of all Lebesgue measurable sets of the real line $R$ is a noncomplete base for the discrete space $R$.

In the last section we give some applications of the complete bases to certain inverse-closed subalgebras of $C(X)$ (called algebras), as a consequence of an important relationship between algebras and bases stated in [10]. To each algebra $A$ on $X$ a certain natural base $\mathscr{Z}(A)$ on $X$ is associated. We find that an algebra $A$ on $X$ is $C(Y)$ for some space $Y$ if and only if $\mathscr{Z}(A)$ is complete. Hence, the examples of noncomplete bases provide examples of algebras that are isomorphic to no $C(Y)$.

Complete bases. When there is no question as to the space $X$, we will write $\omega(X, \mathscr{D})$ (resp. $v(X, \mathscr{D})$ ) as simply $\omega(\mathscr{D})$ (resp. $v(\mathscr{D}))$. The family of all zero-sets in $X$ will be denoted by $Z(X)$. Let $Y$ be a nonempty subset of $X$. The $Q$-closure of $Y$ is the set $Q(Y, X)$ of all points $x \in X$ for which every zero-set in $Z(X)$ containing $x$ has a nonempty intersection with $Y$. The subset $Y$ is $Q$-dense in $X$ if $Q(Y, X)=X$.

The following result about extension of maps is needed.

THEOREM 1. Let $X$ be a dense subspace of a space $T$ and let $\Phi$ be a base on $a$ space $Y$. A continuous map $\varphi: X \rightarrow Y$ has a continuous extension from $T$ to $v(\mathscr{D})$ if and only if for any sequence $\left\{D_{n}\right\}_{n=1}^{\infty}$ of sets in $\mathscr{D}$ such that $\cap_{n=1}^{\infty} D_{n}$ $=\varnothing$, we have $\cap_{n=1}^{\infty} \operatorname{cl}_{T} \varphi^{-1}\left(D_{n}\right)=\varnothing$.

Slight modifications in the proof of Theorem 9.9 in [11] show the result.

A base $\mathscr{D}$ on a space $X$ is said to be complete if it coincides with the family $\hat{\mathscr{D}}=\{Z \cap X: Z \in Z(v(\mathscr{D}))\}$. Since $\mathscr{D}$ is the trace on $X$ of all zero-sets in the Wallman compactification $\omega(X, \mathscr{D})[10$, Theorem 2.2], we have $\mathscr{D} \subset \hat{\mathscr{D}}$. An example of a complete base is $Z(X)$. Later, various examples of noncomplete bases will be given.

The following theorem is the main result.

THEOREM 2. If $\mathscr{D}$ is a base on a space $X$, then $v(\hat{\mathscr{D}})=v(\mathscr{D})$.

PROOF. For convenience we write $E=v(\hat{\mathscr{D}})$ and $F=v(\mathscr{D})$. Since $\mathscr{D} \subset \hat{\mathscr{D}}$, from Theorem 1 there exists a continuous map $\psi$ from $E$ into $F$ whose restriction to $X$ is the identity. It suffices to prove that $\psi$ is a bijection from $E$ onto $F$ whose inverse is continuous.

Let $p$ be an arbitrary point in $F$. Then $\{p\}=\bigcap\{Z \in Z(F): p \in Z\}$. Since $X$ is $Q$-dense in $F$ [1, Theorem 5.16], the family $\{Z \cap X: p \in Z, Z \in$ $Z(F)\}$, is a $\hat{\Phi}$-ultrafilter with the countable intersection property. If $q \in$ $\cap\left\{\operatorname{cl}_{E}(Z \cap X): p \in Z, Z \in Z(F)\right\}$, then $\psi(q)=p$ and therefore $\psi$ is onto. Let us suppose now that $q_{1}$ and $q_{2}$ are distinct points of $E$. There exist $Z_{1}, Z_{2} \in$ OD such that $q_{i} \in \mathrm{cl}_{E} Z_{i}, i=1,2$, and $Z_{1} \cap Z_{2}=\varnothing$. If $Z_{i}^{\prime} \in Z(F)$ and $Z_{i}^{\prime} \cap X=Z_{i}$, then $\psi\left(q_{i}\right) \in \operatorname{cl}_{F}\left(Z_{i}^{\prime} \cap X\right) \subset Z_{i}^{\prime}, i=1,2$. As $X$ is $Q$-dense in $F$ and $Z_{1} \cap Z_{2}=\varnothing$, we have that $Z_{1}^{\prime} \cap Z_{2}^{\prime}=\varnothing$ and $\psi\left(q_{1}\right) \neq \psi\left(q_{2}\right)$. 
On the other hand, from the $Q$-density of $X$ in $F$ it follows that for any sequence $\left\{Z_{n}\right\}_{n=1}^{\infty}$ of sets in $\hat{\mathcal{D}}$ with empty intersection, we have $\cap_{n=1}^{\infty} \operatorname{cl}_{F} Z_{n}$ $=\varnothing$. From Theorem 1, the identity from $X \subset F$ onto $X \subset E$ has a continuous extension from $F$ into $E$, which coincides with the inverse of $\psi$ $[5,0.12]$.

REMARK. In [6] Hager has proved the following interesting result: Let $K$ be a compactification of a space $X$ and let $\mathscr{D}$ be the family $\{Z \cap X: Z \in$ $Z(K)\}$. Then the $Q$-closure $Q(X, K)$ is equivalent to the Wallman realcompactification $v(X, \mathcal{D})$. From Theorem 2 above we obtain $Q(X, K)=$

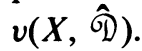

The following is an interesting characterization of the complete bases.

Corollary 2.1. A base $\mathscr{D}$ on a space $X$ is complete if and only if $\beta(v(\mathscr{D}))=\omega(\mathscr{D})$.

Proof. We write $\mathcal{E}=\{Z \cap v(\mathscr{D}): Z \in Z(\omega(\mathscr{D}))\}$. Necessity. Let us prove that $\mathcal{E}=Z(v(\mathscr{D}))$. If $Z \in Z(v(\mathscr{D}))$, then by hypothesis $Z \cap X \in \mathscr{D}$, and there exists $Z^{\prime} \in Z(\omega(\mathscr{D}))$ such that $Z \cap X=Z^{\prime} \cap X$. Since $v(\mathscr{D})$ is the $Q$-closure of $X$ in $\omega(D)$, then $\operatorname{cl}_{v(\mathcal{D})}\left(Z^{\prime} \cap X\right)=Z^{\prime} \cap v(\mathscr{D})$. Therefore $Z^{\prime} \cap$ $v(\mathscr{D}) \subset Z \subset \operatorname{cl}_{v(\mathscr{D})}(Z \cap X) \subset Z^{\prime} \cap v(\mathscr{D})$ and $Z=Z^{\prime} \cap v(\mathscr{D})$. So $Z \in \mathcal{E}$, $\mathcal{E}=Z(v(\mathscr{D}))$ and consequently $\omega(v(\mathscr{D}), \mathcal{E})=\beta(v(\mathscr{D}))$. On the other hand, as $\omega(\mathscr{D})=\omega(v(\mathscr{D}), \mathcal{E})[10$, Theorem 2.9], it follows that $\beta(v(\mathscr{D}))=\omega(\mathscr{D})$.

Sufficiency. By hypothesis $\omega(\mathscr{D})=\omega(v(\mathscr{D}), Z(v(\mathscr{D})))$ and as $\omega(\mathscr{D})=$ $\omega(v(\mathscr{D}), \mathcal{E})$, thus $\mathcal{E}=Z(v(\mathscr{D}))[10$, Corollary 2.3] and therefore $\mathscr{D}=\hat{\mathscr{D}}$.

COROLlaRY 2.2. The following is true: (1) $\hat{D}$ is the largest base of $X$ such that $v(\mathscr{D})=v(\hat{\mathscr{D}})$. (2) $\hat{\mathscr{D}}$ is the smallest complete base in $X$ containing $\mathscr{D}$.

Proof. (1) Let $\mathcal{L}$ be a base in $X$ such that $v(\mathscr{L})=v(\mathscr{D})$. Then $v(\hat{D})=v(\hat{\mathscr{L}})$. From Corollary 2.1 we have $\omega(\hat{\mathscr{D}})=\omega(\hat{\mathcal{L}})$, therefore $\hat{\mathscr{D}}=\hat{\mathcal{L}}$ and $\mathcal{L} \subset \hat{\mathscr{D}}$.

(2) Let $\mathscr{F}$ be a complete base in $X$ containing $\mathscr{D}$. Then $\mathscr{D} \subset \hat{\mathscr{F}}$. From Theorem 1 there exists a continuous map from $v(\hat{\mathscr{F}})$ into $v(\mathscr{D})$ whose restriction to $X$ is the identity. By the definition of $\hat{D}$ we conclude that $\hat{\mathfrak{Q}} \subset \hat{\mathscr{F}}=\mathscr{F}$.

Let $\mathcal{L}$ be a base on a space $X$. The countable covers of $X$ consisting of sets whose complements are members of $\mathcal{L}$ form a base for a (compatible)

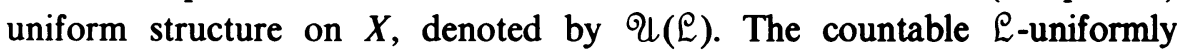
continuous functions in the sense of [3] are precisely those (into $R$ ) which are uniformly continuous in the uniformity $\mathcal{Q}(\mathcal{L})$.

THEOREM 3. Let $\Phi$ be a base on a space $X$. A real-valued function $f$ on $X$ can be continuously extended to $v(\mathcal{D})$ if and only if it is uniformly continuous in the uniformity थ(ดิ)

Proof. Sufficiency. It is a consequence of Theorem 2 above and the theorem of [3]. Necessity. Given $\varepsilon>0$, if $g$ is the continuous extension of $f$ to 
$v(\mathscr{D})$, the sets $V_{n}=\{p \in v(\mathscr{D}): g(p) \leqslant((n-1) / 3) \varepsilon\} \cup\{p \in v(\mathscr{D}): g(p)$ $\geqslant((n+1) / 3) \varepsilon, n=0, \pm 1, \pm 2, \ldots\}$ belong to $Z(v(\mathscr{D}))$. If $O_{n}=X \sim V_{n}$, then $\left\{O_{n}: n=0, \pm 1, \pm 2, \ldots\right\}$ is a countable cover of $X$ by $\hat{\mathscr{D}}$-complements, on each of which the oscillation of $f$ is less than $\varepsilon$, so $f$ is uniformly continuous in the uniformity $\mathscr{Q}(\hat{\mathcal{D}})$.

THEOREM 4. Let $X$ be a realcompact space in which every point is $a G_{\delta}$ and let $X^{*}$ be the set $X$ with a finer completely regular topology. If $\mathscr{B}$ is a base in $X^{*}$ containing $Z(X)$, then $\hat{\mathscr{B}}=Z\left(X^{*}\right)$.

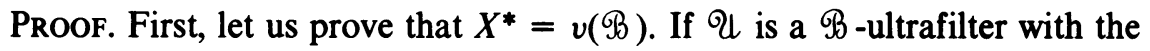
countable intersection property (c.i.p.) the family $\mathscr{F}=\{Z \in Z(X): Z \in \mathscr{Q}\}$ is a prime $Z(X)$-filter with c.i.p. Let $\mathcal{T}$ be the (unique) $Z(X)$-ultrafilter with c.i.p. that contains $\mathscr{F}[11$, Theorem 6.16]. Since $X$ is realcompact there is a point $x_{0}$ in $X$ such that $\left\{x_{0}\right\}=\cap\{Z: Z \in \mathscr{V}\}$. By our hypothesis there exists a decreasing sequence $\left\{Z_{n}\right\}_{n=1}^{\infty}$ of zero-sets in $Z(X)$ such that $X \sim$ int $_{X} Z_{n} \in Z(X), n=1,2, \ldots$, and $\left\{x_{0}\right\}=\cap_{n=1}^{\infty} Z_{n}$. As $\mathscr{F}$ is prime we have that $Z_{n} \in \mathscr{F} \subset \mathcal{U}, n=1,2, \ldots$, and therefore $x_{0} \in \cap\{U: U \in \mathcal{U}\}$. Then $\mathcal{Q}$ is fixed and $X^{*}=v(\Re)$. Since $X^{*}=v\left(Z\left(X^{*}\right)\right)$, from Corollary 2.2 we have $\hat{B}=Z\left(X^{*}\right)$.

The following example shows that the assumption of $Z(X) \subset \mathscr{B}$ is essential.

EXAMPLE. Let $X$ be an uncountable discrete space and let $\mathscr{Q}$ be the family $\{M \subset X: M$ is finite or $X \sim M$ is countable $\}$. Thus $\mathscr{D}$ is a base such that $\omega(\mathscr{D})=v(\mathscr{D})$ is the Alexandroff compactification of $X$. Since $\omega(\mathscr{D})=$ $\beta(v(\mathscr{D})$ ) we have $\mathscr{D}=\hat{\mathscr{D}}$, but $\mathscr{D}$ is not the family $\mathscr{P}(X)$ of all subsets of $X$.

COROLlaRY 4.1. Let $X$ be a realcompact space in which every point is $a G_{\delta}$. Let $\mathscr{Q}$ be $a$ base on $X$ with the discrete topology. If $Z(X) \subset \mathscr{D}$, then $\hat{\mathscr{D}}=\mathscr{P}(X)$.

Then, the $\sigma$-algebra of all Borel sets in $R$ is a noncomplete base of the discrete space $R$, and also, the $\sigma$-algebra of all Lebesgue measurable sets in $R$.

Subalgebras of $C(X)$. As usual, $C(X)$ will denote the ring of all continuous real-valued functions on a space $X$. By an algebra on $X$ is meant a subalgebra of $C(X)$ which separates points and closed sets, contains the constants, and is closed under inversion and uniform convergence. If $A$ is an algebra on $X$ and $\mathscr{Z}(A)=\{Z(f): f \in A\}$, the map $A \rightarrow \mathscr{Z}(A)$ is a one-to-one correspondence between the family of all algebras on $X$ and the family of all bases on $X$ [10, Theorem 4.3]. Moreover, if $A$ is an algebra on $X$ isomorphic to $C(Y)$ for some space $Y$, then $v Y=v(\mathscr{Z}(A))$ and $\beta Y=\omega(\mathscr{Z}(A))[10,4.4]$. Therefore:

TheOREM 5. An algebra $A$ on $X$ is isomorphic to $C(Y)$ for some space $Y$ if and only if $\mathscr{Z}(A)$ is a complete base. 
It is known that an algebra on $X$ needs not to be $C(X)$, nor any $C(Y)$ [6]-[8], [10]. The following result shows that such a situation arises in a very large class of standard function algebras used in Topology and Analysis. It is a consequence of Theorems 4 and 5.

THEOREM 6. Let $X$ be a realcompact space in which every point is a $G_{\delta}$ and let $X^{*}$ be the set $X$ with a finer completely regular topology. If $A$ is an algebra on $X^{*}$ containing $C(X)$, then $A=C\left(X^{*}\right)$ or $A$ is not of the form $C(Y)$.

REMARK. The obvious open problem is to find a constructive method of the completion $\hat{D}$. It has to be noted that since the $\sigma$-algebra of all Lebesgue measurable sets of the real line $R$ is a noncomplete base of the discrete space $R$, many usual set operations have to be disregarded.

\section{REFERENCES}

1. R. A. Alò and H. L. Shapiro, Normal topological spaces, Cambridge Univ. Press, Cambridge, Mass., 1974.

2. H. L. Bentley and S. A. Naimpally, L-realcompactifications as epireflections, Proc. Amer. Math. Soc. 44 (1974), 196-202.

3. A. J. D'Aristotle, A note on $\mathscr{Z}$-realcompactifications, Proc. Amer. Math. Soc. 32 (1972), 615-618.

4. O. Frink, Compactifications and seminormal spaces, Amer. J. Math. 86 (1964), 602-607.

5. L. Gillman and M. Jerison, Rings of continuous functions, The Univ. Series in Higher Math., Van Nostrand, Princeton, N. J., 1960.

6. A. W. Hager, On inverse-closed subalgebras of $C(X)$, Proc. London Math. Soc. 19 (1969), 233-257.

7. M. Henriksen and D. G. Johnson, On the structure of a class of archimedean lattice-ordered algebras, Fund. Math. 50 (1961), 73-94.

8. J. R. Isbell, Algebras of uniformly continuous functions, Ann. of Math. 68 (1958), 96-125.

9. E. F. Steiner, Wallman spaces and compactifications, Fund. Math. 61 (1968), 295-304.

10. A. K. Steiner and E. F. Steiner, Nest generated intersection rings in Tychonoff spaces, Trans. Amer. Math. Soc. 148 (1970), 589-601.

11. M. D. Weir, Hewitt-Nachbin spaces, North-Holland, Amsterdam, 1975.

Catedra de Matematicas iI, Facultad de Ciencias, Buruasot, Valencia, Spain 